Pacific Journal of Mathematics

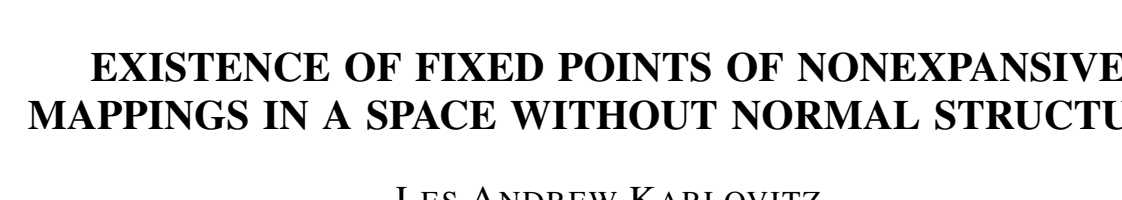




\section{EXISTENCE OF FIXED POINTS OF NONEXPANSIVE MAPPINGS IN A SPACE WITHOUT NORMAL STRUCTURE}

\section{A. Karlovitz}

A mapping $T: C \rightarrow X$ defined on a subset $C$ of a Banach space $X$, with norm $\|\cdot\|$, is said to be nonexpansive if $\| T x-$ $T y\|\leqq\| x-y \|$ for all $x, y \in C$. If $C$ is assumed to be convex and weakly compact and if $T: C \rightarrow C$ then one of the main open questions is whether $T$ has a fixed point in $C$, i.e., whether there exists $x \in C$ so that $T x=x$. If $X$ is reflexive and uniformly convex or, more generally, if $X$ is reflexive and has normal structure then the answer is affirmative. Our purpose is to give an example of a classical reflexive space which does not have normal structure and for which the answer is nevertheless affirmative.

Nonexpansive mappings have played an important role in recent developments of nonlinear functional analysis (e.g., Browder [1], deFigueiredo [3]). The analysis of the uniformly convex and normal structure cases is due to Browder [2], Göhde [4] and Kirk [6].

Let $X_{J}$ be the space $l_{2}$ renormed according to

$$
\|x\|=\max \left\{\|x\|_{\infty},\|x\|_{2} / \sqrt{2}\right\}
$$

where $\|\cdot\|_{\infty}$ denotes the $l_{\infty}$ norm and $\|\cdot\|_{2}$ the $l_{2}$ norm. This space originates with R. C. James. A space is said to have normal structure if for each bounded convex subset $C$ consisting of more than one point there is a point $w \in C$ so that $\sup \{\|w-y\|: y \in C\}<$ $\sup \{\|x-y\|: x, y \in C\}$. The set $\left\{x: x=x(i), x(i) \geqq 0,\|x\|_{2} \leqq 1\right\}$ in $X_{J}$ contains no such point $w$.

THEOREM. Let $C$ be a bounded closed convex subset of $X_{\mathrm{J}}$. Let $T: C \rightarrow C$ be nonexpansive. Then $T$ has a fixed point in $C$.

One of the main tools in the proof is the following general lemma which has been used in Karlovitz [5].

Lemma. Let $X$ be a Banach space. Let $C_{0}$ be a weakly compact convex subset of $X$. Let $T: C_{0} \rightarrow C_{0}$ be nonexpansive. Suppose that $C_{0}$ is minimal in the sense that it contains no proper closed convex subset which is 
invariant under $T$. Let $\left\{x_{n}\right\}$ be a sequence of approximate fixed points, i.e., $x_{n} \in C_{0}$ and $\left\|T x_{n}-x_{n}\right\| \rightarrow 0$. Then for each $x \in C_{0}$

$$
\lim _{n}\left\|x-x_{n}\right\|=\operatorname{diameter} C_{0} \text {. }
$$

If $X$ is reflexive then a standard argument establishes the existence of a subset $C_{0} \subset C$ which is minimal in the sense of the lemma. If $C_{0}$ consists of more than one point, another standard argument yields a sequence of approximate fixed points in $C_{0}$. It follows from (1) that $X$ does not have normal structure. Hence if $X$ does have normal structure then $C_{0}$ is necessarily a singleton and thus a fixed point of $T$. This is an alternate proof of the known result. In order to use the lemma to show that in $X_{J} C_{0}$ is necessarily a singleton, we need to make more explicit use of the nonexpansiveness of $T$ and of the geometry of $X_{J}$. While some modest generalizations will become apparent, the implications for the general reflexive case are not yet clear.

Proof of the lemma. Choose $y \in C_{0}$ and let $s=$ limsup $\left\|y-x_{n}\right\|$. Let $D=\left\{x: x \in C_{0}\right.$, limsup $\left.\left\|x-x_{n}\right\| \leqq s\right\}$, which is nonempty closed and convex. It is also invariant under $T$. For

$$
\left\|T x-x_{n}\right\| \leqq\left\|T x-T x_{n}\right\|+\left\|T x_{n}-x_{n}\right\| \leqq\left\|x-x_{n}\right\|+\left\|T x_{n}-x_{n}\right\|
$$

and $\left\|T x_{n}-x_{n}\right\| \rightarrow 0$. By the minimality of $C_{0}, D=C_{0}$. Extract a subsequence $\left\{x_{n^{\prime}}\right\}$ so that $\lim \left\|y-x_{n^{\prime}}\right\|=s^{\prime}$ exists. Suppose that there exists $z \in C_{0}$ and a subsequence $\left\{x_{n^{\prime \prime}}\right\}$ of $\left\{x_{n^{\prime}}\right\}$ so that $\lim \left\|z-x_{n^{\prime \prime}}\right\|=t$. Let $E=\left\{x: x \in C_{0}, \lim \sup \left\|x-x_{n^{\prime \prime}}\right\| \leqq \min \left\{t, s^{\prime}\right\}\right\}$. Repeating the argument, we find $E=C_{0}$. Hence $y, z \in E$, and so $t=s^{\prime}$. Thus for each $x \in C_{0}$, $\lim \left\|x-x_{n^{\prime}}\right\|$ exists and equals $s^{\prime}$.

We complete the proof by showing that $s^{\prime}=r=\operatorname{diam} C_{0}$. From this it follows that $\left\|y-x_{n^{\prime}}\right\| \rightarrow r$ whenever $\left\{\left\|y-x_{n^{\prime}}\right\|\right\}$ converges. Whence, by boundedness, $\left\|y-x_{n}\right\| \rightarrow r$ for the entire sequence. By repeating the argument above with $\left\{x_{n}\right\}$ replaced by the entire sequence $\left\{x_{n}\right\}$, we shall have proved (1).

To this end, consider $F=\left\{u: u \in C_{0},\|u-x\| \leqq s^{\prime}\right.$ for each $\left.x \in C_{0}\right\} . \quad F$ is nonempty because we can extract a weakly convergent subsequence, again denoted by $\left\{x_{n^{\prime}}\right\}$, with limit $z$. Since $\left\|x-x_{n^{\prime}}\right\| \rightarrow s^{\prime}$ for each $x \in C_{0}$ it follows that $\|x-z\| \leqq s^{\prime}$ for each $x \in C_{0}$; hence $z \in F$. Now if $s^{\prime}<r$ then $F \varsubsetneqq C_{0}$. However, this contradicts the minimality of $C_{0}$ because $F$ is invariant under $T$. To see the latter, we first note that as a consequence of minimality closed convex hull $\left(T C_{0}\right)=$ $C_{0}$. Hence if $u$ is an arbitrary element of $C_{0}$ then for given $\epsilon>0$ we can choose $v=\sum_{l=1}^{k} \lambda_{i} T x_{i} \quad$ with $x_{i} \in C_{0}, \quad \lambda_{\imath}>0, \quad \Sigma \lambda_{i}=1$ and $\|u-v\| \leqq$ $\epsilon$. Choose arbitrary $w$ in $F$. Then 


$$
\begin{aligned}
\|T w-u\| & \leqq\|T w-v\|+\|v-u\| \\
& \leqq \Sigma \lambda_{\imath}\left\|T w-T x_{\imath}\right\|+\|v-u\| \\
& \leqq \Sigma \lambda_{i}\left\|w-x_{i}\right\|+\|v-u\| \leqq s^{\prime}+\epsilon .
\end{aligned}
$$

Since $\epsilon>0$ and $u \in C_{0}$ are arbitrary this shows $T w \in F$. Thus $F$ is invariant under $T$ and hence $s^{\prime}=r$. This finishes the proof.

Proof of the theorem. Given $x \in X_{J}$ we represent its components by $x(j), j=1,2, \cdots$. Since $X_{J}$ is a renorming of $l_{2}$ there exists a component $x(j)$ so that $\|x\|_{\infty}=|x(j)|$.

By a standard Zorn's lemma argument there exists a closed convex subset $C_{0}$ of $C$ which is invariant under $T$ and minimal in the sense of the lemma. We propose to show that $C_{0}$ consists of a single point. By invariance this is then a fixed point of $T$.

We proceed by contradiction. Suppose that $C_{0}$ consists of more than one point. We may assume without loss of generality that $0 \in C_{0}$, and we let diameter $C_{0}=r>0$. For each $s, 0<s<1$, we define $T_{s}=(1-s) T$. Clearly $T_{s}: C_{0} \rightarrow C_{0}$ and it is a strict contraction. Hence by the Banach contraction principle there exists a unique $x_{s} \in C_{0}$ so that $T_{s} x_{s}=x_{s}$. Thus

$$
T x_{s}=(1-s)^{-1} x_{s}, \quad \text { for } \quad 0<s<1 .
$$

By the minimality of $C_{0}, x_{s} \neq 0$. The desired contradiction results from a study of the points $x_{s}$. Several propositions are needed.

Proposition 1. For each $x \in C_{0}, \lim _{s \rightarrow 0}\left\|x-x_{s}\right\|_{\infty}=r$.

Proof. By contradiction. Suppose that for some $x \neq 0 \in C_{0}$ and sequence $\left\{x_{s_{n}}\right\}$ with $s_{n} \rightarrow 0$, denoted by $\left\{x_{n}\right\},\left\|x-x_{n}\right\|_{\infty} \leqq r-\delta, \delta>$ 0 . Since $\left\|0-x_{n}\right\|_{\infty} \leqq \operatorname{diam} C_{0}=r$ it follows that $\left\|x / 2-x_{n}\right\|_{\infty} \leqq r-\delta / 2$ for all $n$. By the uniform convexity of $\|\cdot\|_{2}$, it follows from $\left\|x-x_{n}\right\|_{2} / \sqrt{2}$, $\left\|0-x_{n}\right\|_{2} / \sqrt{2} \leqq \operatorname{diam} C_{0}=r$ that $\left\|x / 2-x_{n}\right\|_{2} / \sqrt{2} \leqq r-\tau$ for some $\tau>$ 0. Hence, $\left\|x / 2-x_{n}\right\| \leqq r-\min \{\tau, \delta / 2\}$ for all $n$, which contradicts (1) because $\left\|T x_{n}-x_{n}\right\|=s_{n}\left(1-s_{n}\right)^{-1}\left\|x_{n}\right\| \rightarrow 0$.

Proposition 2. For each $s, 0<s<1, \lim _{t \rightarrow s}\left\|x_{t}-x_{s}\right\|=0$.

Proof. We denote $x_{s}$ by $x$ and $x_{t}$ by $y$. Suppose that $\|x-y\|=$ $\|x-y\|_{\infty}=|x(k)-y(k)|$. By nonexpansiveness

$$
\left|(1-s)^{-1} x(k)-(1-t)^{-1} y(k)\right| \leqq|x(k)-y(k)| .
$$


For $s \neq t$ it readily follows that $\operatorname{sgn} x(k)=\operatorname{sgn} y(k)=\sigma= \pm 1$. Suppose that $0<t<s$. If $\sigma x(k)>\sigma y(k)$ then

$$
(1-s)^{-1} \sigma x(k)-(1-t)^{-1} \sigma y(k)>(1-t)^{-1}(\sigma x(k)-\sigma y(k)),
$$

contradicting (2). Hence $\sigma y(k) \geqq \sigma x(k)$. If $(1-t)^{-1} \sigma y(k)-$ $(1-s)^{-1} \sigma x(k) \geqq 0$ then, by $(2), \quad(1-s) t(1-t)^{-1} s^{-1} \sigma y(k) \leqq \sigma x(k) \leqq$ $\sigma y(k)$. If $(1-s)^{-1} \sigma x(k)-(1-t)^{-1} \sigma y(k) \geqq 0$ then, directly, $(1-s)$ $(1-t)^{-1} \sigma y(k) \leqq \sigma x(k) \leqq \sigma y(k)$. If $s<t<1$, analogous inequalities are derived. It follows that

$$
|x(k)-y(k)| \leqq \begin{cases}(s-t) s^{-1}(1-t)^{-1}|y(k)|, & 0<t<s \\ (t-s) t^{-1}(1-s)^{-1}|x(k)|, & s<t<1\end{cases}
$$

Hence if $\left\|x_{t}-x_{s}\right\|=\left\|x_{s}-x_{t}\right\|_{\infty}$ and $s / 2<t<(1+s) / 2$,

$$
\left\|x_{s}-x_{t}\right\| \leqq A|s-t|, \text { for some } A=A(s)>0 .
$$

Now suppose that $\|x-y\|=\|x-y\|_{2} / \sqrt{2}$. By nonexpansiveness

$$
\left\|(1-s)^{-1} x-(1-t)^{-1} y\right\|_{2} \leqq\|x-y\|_{2} \text {. }
$$

We divide the positive integers according to:

$$
I_{1}=\left\{i:\left|(1-s)^{-1} x(i)-(1-t)^{-1} y(i)\right| \leqq|x(i)-y(i)|\right\}
$$

and

$$
I_{2}=\left\{i:\left|(1-s)^{-1} x(i)-(1-t)^{-1} y(i)\right|>|x(i)-y(i)|\right\} .
$$

Then

(5) $\sum_{I_{2}}\left[\left((1-s)^{-1} x(i)-(1-t)^{-1} y(i)\right)^{2}-(x(i)-y(i))^{2}\right] \leqq \sum_{I_{1}}(x(i)-y(i))^{2}$.

By definition, (2) holds for $k \in I_{1}$. We can deduce, as above, that (3) holds. Whence, for $s / 2<t<(1+s) / 2$

$$
\sum_{I_{1}}(x(i)-y(i))^{2} \leqq B(s-t)^{2} \quad \text { for some } \quad B=B(s)>0
$$

We note the identity.

$$
(1-t)^{-1} y(i)-(1-s)^{-1} x(i)=(1-s)^{-1}(y(i)-x(i)-\gamma(s, t) y(i)),
$$


where $\gamma(s, t)=(s-t)(1-t)^{-1}$. Substitution into (5) yields

$$
\begin{aligned}
\sum_{I_{2}}\left[(1-s)^{-2}((y(i)-x(i))-\gamma(s, t) y(i))^{2}-(x(i)-y(i))^{2}\right] & \\
& \leqq \sum_{I_{1}}(x(i)-y(i))^{2} .
\end{aligned}
$$

By the Schwarz inequality and some simple manipulation

$$
\sum_{I_{2}}(x(i)-y(i))^{2} \leqq s^{-1}(1-s)^{2} \cdot \sum_{I_{1}}(x(i)-y(i))^{2}+2 s^{-1} \gamma(s, t)\|y\|_{2}\|x-y\|_{2} .
$$

Combining this with (6) we find that if $\left\|x_{s}-x_{t}\right\|=\left\|x_{s}-x_{t}\right\|_{2} / \sqrt{2}$ and $s / 2<t<(1+s) / 2$ then

$$
\left\|x_{s}-x_{t}\right\| \leqq K(s-t)^{1 / 2} \text { for some } \quad K=K(s)>0 .
$$

The proposition now follows from (4) and (7).

For each positive integer $i$ and $\epsilon>0$ we introduce the notation:

$$
A^{\epsilon}(i)=\left\{s: 0<s<1,\left|x_{s}(i)\right| \geqq r-\epsilon\right\} \quad \text { and } \quad \alpha^{\epsilon}(i)=\inf A^{\epsilon}(i) \text {. }
$$

Proposition 3. For each positive integer $i$ and $\epsilon, 0<\epsilon \leqq r / 4$, there exists $s_{1}, 0<s_{1}<1$, with the property that for each $s, 0<s \leqq s_{1}$, there exists a positive integer $k(s)$ such that $k(s) \neq i$ and $\left|x_{s}(k(s))\right| \geqq r-\epsilon$.

Proof. If $A^{\epsilon}(i)=\varnothing$ this follows from Proposition 1 with $x=$ 0 . Otherwise choose $s_{0} \in A^{\epsilon}(i)$. Let $\epsilon_{1}=\min \left\{s_{0}\left(1-s_{0}\right)^{-1}(r-\epsilon), \epsilon / 2\right.$, $\left.r s_{0} / 2, s_{0}\left(1-s_{0}\right)^{-1} \epsilon / 2\right\}$. By Proposition 1 choose $s_{1}$ so that $\left\|x_{s_{0}}-x_{s}\right\|_{\infty} \geqq$ $r-\epsilon_{1}$ for all $0<s \leqq s_{1}$. By virtue of $T x_{s 0}=\left(1-s_{0}\right)^{-1} x_{s 0} \in C_{0}$ we have $\left\|x_{s 0}\right\|_{\infty} \leqq r\left(1-s_{0}\right)$. Choose $s, \cdot 0<s \leqq s_{1}$. Suppose that $\operatorname{sgn} x_{s_{0}}(i)=$ $\operatorname{sgn} x_{s}(i)$ or $x_{s}(i)=0$. Then from $3 r / 4 \leqq\left|x_{\text {so }}(i)\right| \leqq r\left(1-s_{0}\right)$ we deduce $\left|x_{s o}(i)-x_{s}(i)\right| \leqq r-r s_{0}, r / 4<r-\epsilon_{1}$. If $\operatorname{sgn} x_{s o}(i)=-\operatorname{sgn} x_{s}(i)$ then

$$
\begin{aligned}
r \geqq & \left\|T x_{s 0}-T x_{s}\right\| \geqq\left|\left(1-s_{0}\right)^{-1} x_{s 0}(i)-(1-s)^{-1} x_{s}(i)\right|>\left|x_{s 0}(i)-x_{s}(i)\right| \\
& +s_{0}\left(1-s_{0}\right)^{-1}\left|x_{s 0}(i)\right| \geqq\left|x_{s 0}(i)-x_{s}(i)\right| \\
& +s_{0}\left(1-s_{0}\right)^{-1}(r-\epsilon) \geqq\left|x_{s 0}(i)-x_{s}(i)\right|+\epsilon_{1},
\end{aligned}
$$

and hence $\left|x_{s o}(i)-x_{s}(i)\right|<r-\epsilon_{1}$. Thus there exists a positive integer $j \neq i$ so that $\left\|x_{s_{0}}-x_{s}\right\|_{\infty}=\left|x_{s 0}(j)-x_{s}(j)\right| \geqq r-\epsilon_{1}$. We assert that $k(s)=j$ satisfies the proposition. If $\operatorname{sgn} x_{s 0}(j)=\operatorname{sgn} x_{s}(j)$ then $r-\epsilon_{1} \leqq$ $\left|x_{s 0}(j)-x_{s}(j)\right|<\max \left\{\left|x_{s 0}(j)\right|,\left|x_{s}(j)\right|\right\}$. Since $\left|x_{s 0}(j)\right| \leqq r\left(1-s_{0}\right)<r-\epsilon_{1}$ it follows that $\left|x_{s}(j)\right|>r-\epsilon_{1}>r-\epsilon$, as desired. If $\operatorname{sgn} x_{s o}(j) \neq \operatorname{sgn} x_{s}(j)$, then 


$$
\begin{aligned}
r \geqq & || T x_{s o}-T x_{s} \| \geqq\left|\left(1-s_{0}\right)^{-1} x_{s 0}(j)-(1-s)^{-1} x_{s}(j)\right|=\left(1-s_{0}\right)^{-1}\left|x_{s o}(j)\right| \\
& +(1-s)^{-1}\left|x_{s}(j)\right| \geqq\left|x_{s 0}(j)\right|+\left|x_{s}(j)\right|+s_{0}\left(1-s_{0}\right)^{-1}\left|x_{s 0}(j)\right| \\
= & \left|x_{s o}(j)-x_{s}(j)\right|+s_{0}\left(1-s_{0}\right)^{-1}\left|x_{s o}(j)\right| \geqq r-\epsilon_{1}+s_{0}\left(1-s_{0}\right)^{-1}\left|x_{s 0}(j)\right| .
\end{aligned}
$$

Hence $\left|x_{s 0}(j)\right| \leqq s_{0}^{-1}\left(1-s_{0}\right) \epsilon_{1}$. So $\left|x_{s 0}(j)-x_{s}(j)\right| \geqq r-\epsilon_{1}$ implies $\left|x_{s}(j)\right| \geqq$ $r-\epsilon_{1}-s_{0}^{-1}\left(1-s_{0}\right) \epsilon_{1} \geqq r-\epsilon_{1}-\epsilon / 2 \geqq r-\epsilon$, as desired. Since $s \leqq s_{1}$ was arbitrarily chosen this finishes the proof.

Proposition 4. Suppose $\alpha^{\epsilon}(i)=\alpha^{\delta}(j)=0$ for some $\epsilon, \delta$ with $0<\epsilon$, $\delta \leqq r / 64$. Then $i=j$.

Proof. For $i$ and $\epsilon$ we choose $s_{1}$ according to Proposition 3. Thus if $s \in A^{\epsilon}(i)$ and $s \leqq s_{1}$ then $\left|x_{s}(m)\right| \geqq r-\epsilon$ for $m=i, k(s)$. Since $i \neq k(s)$ and $\left\|x_{s}\right\|_{2}^{2} \leqq 2 r^{2}$ we readily find that $\left|x_{s}(m)\right| \leqq r / 4$ for $m \neq i, k(s)$. By Proposition 1 we choose $s_{2}, s_{3} \in A^{\epsilon}(i), s_{2}, s_{3}<s_{1}$, so that $\left\|x_{s_{p}}-x_{s_{q}}\right\|_{\infty} \geqq r-\epsilon$ for $p \neq q$ and $p, q=1,2,3$. Now suppose that $k\left(s_{1}\right)=$ $k\left(s_{2}\right)$. Then $\left|x_{s_{1}}(m)-x_{s_{2}}(m)\right| \leqq\left|x_{s_{1}}(m)\right|+\left|x_{s_{2}}(m)\right| \leqq r / 2<r-\epsilon$ for $m \neq i, k\left(s_{1}\right)$. Moreover, $\operatorname{sgn} x_{s_{1}}(i)=\operatorname{sgn} x_{s 2}(i)$; otherwise $\left\|x_{s_{1}}-x_{s_{2}}\right\|_{\infty} \geqq$ $\left|x_{s_{1}}(i)\right|+\left|x_{s_{2}}(i)\right| \geqq 2 r-2 \epsilon>r$. Thus $\left|x_{s_{1}}(i)-x_{s_{2}}(i)\right| \leqq r-(r-\epsilon)=\epsilon$. By the same argument $\left|x_{s_{1}}\left(k\left(s_{1}\right)\right)-x_{s_{2}}\left(k\left(s_{1}\right)\right)\right| \leqq \epsilon$. Thus $\left|x_{s_{1}}(i)-x_{s_{2}}(i)\right|<r-\epsilon$ for all positive integers $i$, which is a contradiction. Hence $k\left(s_{1}\right) \neq k\left(s_{2}\right)$. Similarly $k\left(s_{3}\right) \neq k\left(s_{1}\right), k\left(s_{2}\right)$. Now if $i \neq j$ we repeat the argument and find $t_{1}, t_{2}, t_{3} \in A^{\delta}(j)$ so that $\left|x_{t_{p}}(j)\right|,\left|x_{t_{p}}\left(k\left(t_{p}\right)\right)\right| \geqq r-\delta$, $p=1,2,3$ and so that $j, k\left(t_{1}\right), k\left(t_{2}\right)$ and $k\left(t_{3}\right)$ are disjoint. Thus we can find $s_{q}$ and $t_{p}$ so that $\left\{i, k\left(s_{q}\right)\right\} \cap\left\{j, k\left(t_{p}\right)\right\}=\varnothing$. Then from $\left|x_{s_{q}}(i)\right|$, $\left|x_{s_{q}}\left(k\left(s_{q}\right)\right)\right| \geqq r-\epsilon,\left|x_{t_{p}}(j)\right|,\left|x_{t_{p}}\left(k\left(t_{p}\right)\right)\right| \geqq r-\delta$ and $\left\|x_{t_{p}}\right\|_{2},\left\|x_{s_{q}}\right\|_{2} \leqq \sqrt{2} r$ it follows that $\left\|x_{s_{q}}-x_{t_{p}}\right\|_{2} / \sqrt{2}>r$ which contradicts $x_{s_{q}}, x_{t_{p}} \in C_{0}$. Hence $i=j$.

Completion of the proof of the theorem. Let $\epsilon=r / 128$. If there exists a positive integer $i$ so that $\alpha^{\epsilon}(i)=0$, let $i_{0}=i$. Otherwise $\alpha^{\epsilon}(i)>0$ for each $i$ and we let $i_{0}=1$. Apply Proposition 3 to find $s_{1}=s_{1}\left(i_{0}, \epsilon\right)$. In the sequel the positive integers $k(\cdot)$ will be those given by the proposition for this $s_{1}$. Denote $k\left(s_{1}\right)$ by $k_{1}$. Let $s_{2}=\alpha^{\epsilon}\left(k_{1}\right)$. If $\alpha^{\epsilon}\left(i_{0}\right)=0$ it follows from $i_{0} \neq k_{1}$ and Proposition 4 that $s_{2}>0$; otherwise $s_{2}>0$ by hypothesis. By Proposition $2\left\|x_{s_{2}-\mu}-x_{s_{2}}\right\| \rightarrow 0$ as $\mu \rightarrow 0$. Hence we can choose $\mu>0$ so that $r-2 \epsilon \leqq\left|x_{s_{2}-\mu}\left(k_{1}\right)\right|$. Since $s_{2}-\mu<s_{2}<s_{1}, \quad k\left(s_{2}-\mu\right)$ is well defined. Since $s_{2}-\mu<\alpha^{\epsilon}\left(k_{1}\right)$, $\left|x_{s^{2}-\mu}\left(k_{1}\right)\right|<r-\epsilon$; and hence $k\left(s_{2}-\mu\right) \neq k_{1}$. Denote $x_{s_{2}-\mu}$ by $y$ and $k\left(s_{2}-\mu\right)$ by $k_{2}$. Thus $\left|y\left(k_{1}\right)\right|,\left|y\left(k_{2}\right)\right| \geqq r-2 \epsilon$. Since $k_{1}, k_{2} \neq i_{0}$, reasoning as above, $\alpha^{\epsilon}\left(k_{1}\right), \alpha^{\epsilon}\left(k_{2}\right)>0$. Hence we can choose $s_{3}$, $0<s_{3}<\alpha^{\epsilon}\left(k_{1}\right), \alpha^{\epsilon}\left(k_{2}\right)$. Then $\left|x_{s_{3}}\left(k_{1}\right)\right|,\left|x_{s 3}\left(k_{2}\right)\right|<r-\epsilon$, and hence $k_{3}=$ $k\left(s_{3}\right) \neq k_{1}, k_{2}$. Repeating the argument we find $z=x_{s_{3}-\eta}, \eta>0$, and $k_{4}=k\left(s_{3}-\eta\right) \neq k_{3} \quad$ so that $\quad\left|z\left(k_{3}\right)\right| \geqq r-2 \epsilon \quad$ and $\quad\left|z\left(k_{4}\right)\right| \geqq$ 
$r-\epsilon$. Moreover, $s_{3}-\eta<\alpha^{\epsilon}\left(k_{1}\right), \alpha^{\epsilon}\left(k_{2}\right)$, hence $k_{4} \neq k_{1}, k_{2}$. Thus $k_{1}$, $k_{2}, k_{3}$ and $k_{4}$ are disjoint. Hence from $\|y\|,\|z\| \leqq r$ and $\left|y\left(k_{1}\right)\right|,\left|y\left(k_{2}\right)\right|$, $\left|z\left(k_{3}\right)\right|,\left|z\left(k_{4}\right)\right| \geqq r-2 \epsilon$ we readily calculate $\|y-z\|_{2} / \sqrt{2}>r$ which contradicts $y, z \in C_{0}$. This contradiction proves that $C_{0}$ cannot consist of more than one point and finishes the proof of the theorem.

Added in proof. Additional applications of the lemma as well as some of its points of contact with work of M. Edelstein will be discussed elsewhere. P. M. Fitzpatrick has informed us that he independently developed the lemma.

\section{REFERENCES}

1. F. E. Browder, Nonlinear mappings of nonexpansive and accretive type in Banach spaces, Bull. Amer. Math. Soc., 73 (1967), 875-881.

2. - Nonexpansive nonlinear operators in a Banach space, Proc. Nat. Acad. Sci. U.S.A., 54 (1965), 1041-1044.

3. D. G. deFigueiredo, Topics in nonlinear functional analysis, Lecture Notes, University of Maryland, 1967.

4. D. Göhde, Zum Prinzip der kontraktiven Abbildungen, Math. Nachr., 30 (1966), 251-258.

5. L. A. Karlovitz, Some fixed point results for nonexpansive mappings, Proceedings of a Seminar on Fixed Points, Dalhousie University 1975, to appear.

6. W. A. Kirk, A fixed point theorem of mappings which do not increase distance, Amer. Math. Monthly, 76 (1965), 1004-1006.

Received June 4, 1976. Research supported in part by the National Science Foundation under Grant GP-20555.

UNIVERSITY OF MARYLAND 




\section{Pacific Journal of Mathematics}

\section{Vol. 66, No. $1 \quad$ November, 1976}

Helen Elizabeth. Adams, Factorization-prime ideals in integral domains ............ Patrick Robert Ahern and Robert Bruce Schneider, The boundary behavior of Henkin's kernel.

Daniel D. Anderson, Jacob R. Matijevic and Warren Douglas Nichols, The Krull

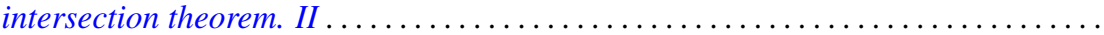

Efraim Pacillas Armendariz, On semiprime P.I.-algebras over commutative regular

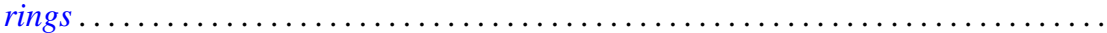

Robert H. Bird and Charles John Parry, Integral bases for bicyclic biquadratic fields

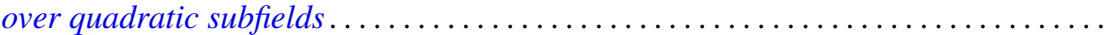

Tae Ho Choe and Young Hee Hong, Extensions of completely regular ordered

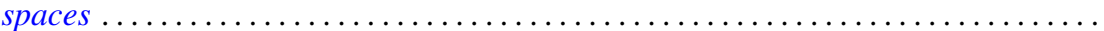

John Dauns, Generalized monoform and quasi injective modules ...............

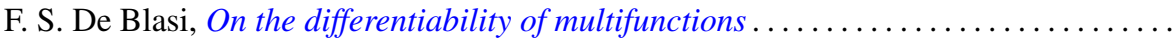

Paul M. Eakin, Jr. and Avinash Madhav Sathaye, R-endomorphisms of $R[[X]]$ are

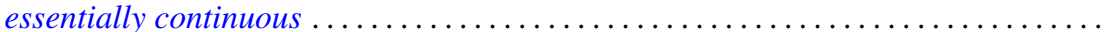

Larry Quin Eifler, Open mapping theorems for probability measures on metric

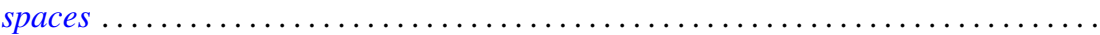

Garret J. Etgen and James Pawlowski, Oscillation criteria for second order self adjoint

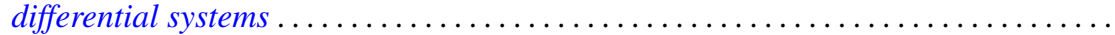

Ronald Fintushel, Local $S^{1}$ actions on 3-manifolds .

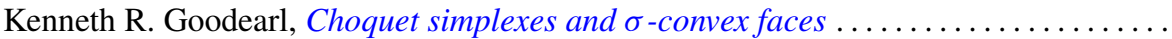

John R. Graef, Some nonoscillation criteria for higher order nonlinear differential

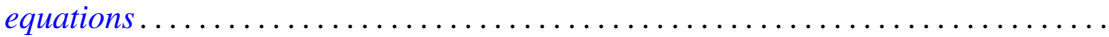

Charles Henry Heiberg, Norms of powers of absolutely convergent Fourier series: an example.

Les Andrew Karlovitz, Existence of fixed points of nonexpansive mappings in a space

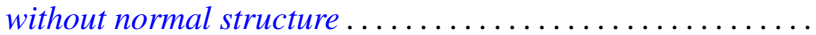

Gangaram S. Ladde, Systems of functional differential inequalities and functional

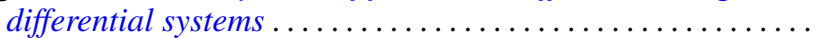

Joseph Michael Lambert, Conditions for simultaneous approximation and interpolation with norm preservation in $C[a, b]$.

Ernest Paul Lane, Insertion of a continuous function.

Robert F. Lax, Weierstrass points of products of Riemann surfaces .

Dan McCord, An estimate of the Nielsen number and an example concerning the Lefschetz fixed point theorem...

Paul Milnes and John Sydney Pym, Counterexample in the theory of continuous functions on topological groups...

Peter Johanna I. M. De Paepe, Homomorphism spaces of algebras of holomorphic functions

Judith Ann Palagallo, A representation of additive functionals on $L^{p}$-spaces,

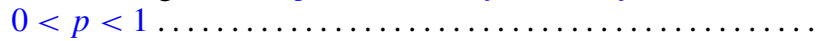

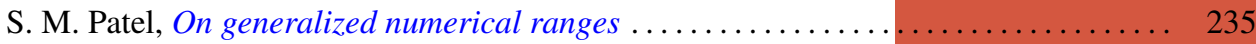

Thomas Thornton Read, A limit-point criterion for expressions with oscillatory

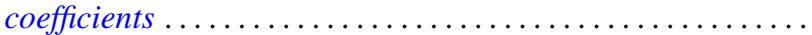

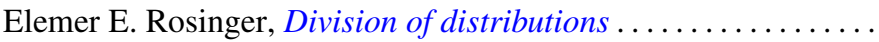

Peter S. Shoenfeld, Highly proximal and generalized almost finite

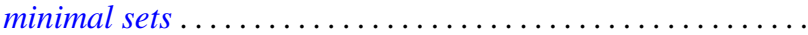

R. Sirois-Dumais and Stephen Willard, Quotient-universal sequential spaces

Robert Charles Thompson, Convex and concave functions of singular values of matrix sums....

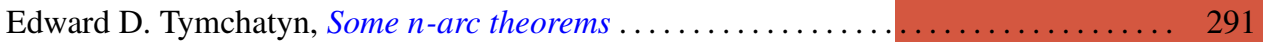

